\title{
Growth of Vertically Aligned InGaN Nanowires
}

\author{
Fangliang Gao* \\ Institute of Semiconductor of Science and Technology, South China Normal University, China
}

*Corresponding author: Fangliang Gao, Institute of Semiconductor of Science and Technology, South China Normal University, Guangzhou 510631, PR China.

Received Date: August 19, 2019

Published Date: August 22, 2019

\begin{abstract}
InGaN nanowires (NWs) have attracted a lot of research interest in the past decade. They contain both the intrinsic properties of InGaN materials, and some unique properties induced by the NW structures. This article reviews the growth methods to obtain InGaN NWs, with discussions on different epitaxy methods.
\end{abstract}

\section{Discussion}

One-dimensional (1D) semiconducting materials have attracted considerable attention for future device applications, such as nanophotonic and nano electronic devices [1]. Group III-nitride nanowires (NWs), InGaN NW is of tunable bandgap (0.7-3.4 eV), high carrier mobility, and excellent optical absorption, which have demonstrated great potentials for the high-performance photodetectors, light-emitting diodes, and solar cells. The optoelectronic performance of InGaN NW devices is highly associated with the NW morphologies [2]. For instance, poorly defined morphology such as a conical shape may cause a significant Fermi level fluctuation along the NW axial direction, leading to undesirable properties of the NWs as well as the poor performance of the related devices. Therefore, the high quality and vertically aligned InGaN NWs is one of the key points for the device applications of the InGaN NWs. The InGaN NW growth methods are reviewed briefly in the subsequent sections.

Recently numerous achievements for well-aligned and uniform InGaN NWs have been obtained through catalyst-assisted "vaporliquid-solid" (VLS) growth, self-catalytic VLS growth and selective area growth (SAG) techniques.

The growth of InGaN NRs is enabled by the VLS growth mode, where liquid or solid metal particles (e.g., $\mathrm{Au}$ ) is used as catalysts. And a high V/III growth condition is conducive to avoiding the formation of stacking dislocation of InGaN NWs and improving the crystal quality. In addition, $\mathrm{Ni}[3], \mathrm{Ni}+\mathrm{Au}[4,5]$ nanoparticles are also widely used as catalysts. As for catalyst-assisted VLS growth, using a metal nanoparticle catalyst, as-grown NWs often differ in their crystallographic orientation and tend to tilt, random, and branch during the growth process. And the random orientation of NW makes the device fabrication difficult. Moreover, catalyst contamination such as $\mathrm{Au}$ or $\mathrm{Ni}$ nanoparticles can result in undesirable mid-gap electronic states in devices, thus markedly degrading the performance of the devices.

To catalyst assisted VLS growth mode, impurity incorporation into NW due to the use of a catalyst may degrade device performance. Therefore, InGaN NW fabrication without any metal catalyst is preferred for device development [6]. As an alternative, the self-catalytic VLS mode has been performed for growing InGaN NWs. However, the orientation of the InGaN NWs is still random or tile. Furthermore, to better control the geometry of InGaN NW, the InGaN NWs can be derived from the high nucleation density of the NWs and the radial growth have been proposed previously by several groups. Thus, the well-separated and vertically aligned NWs can be achieved by enhancing the axial growth rate and suppressing the growth in the radial direction, simultaneously. Of course, the nucleation density of NWs should also be controlled and optimized. In particular, the enhancement of adatom diffusion can lead to a lower nucleation density and be beneficial to improve the axial growth rate, the geometry (the cross-sectional size, height, and shape) of the grown NWs is highly uniform. Meanwhile, the crystal quality is quite high [7].

SAG of InGaN NWs on patterned substrates is proposed to obtain uniformly arranged and uncoalesced NWs. The InGaN NWs are prepared on dielectric-masked nanoholes with selective area 
metalorganic chemical vapor deposition (MOCVD). To overcome the tendency for random nucleation of InGaN at low temperatures, a pulsed growth procedure was introduced to enhance the diffusion length of $\mathrm{Ga}$ adatoms on $\mathrm{SiO}_{2}$, resulting in good selectivity at typical temperature ranges for InGaN [8]. However, the patterning of the mask layers for a subsequent SAG makes this approach more demanding and complex from a technological point of view, as well as expensive from a cost perspective. For the SAG of NWs, a series of extremely complicated and costly techniques such as e-beam lithography and focused ion beam milling are employed, leading to the high cost and low efficiency for fabricating NW arrays [7,9].

\section{Conclusion}

In summary, we have briefly reviewed the method of InGaN NWs growth, including catalyst assisted VLS, self-catalytic VLS, and SAG growth. Therefore, the growth method of InGaN NWs with vertically aligned, less cost and without any metal catalyst is preferred development.

\section{Acknowledgement}

None.

\section{Conflict of Interest}

No conflict of interest.

\section{References}

1. Sarwar A, Carnevale SD, Yang F, Kent TF, Jamison JJ, et al. (2015) Semiconductor Nanowire Light-Emitting Diodes Grown on Metal: A
Direction Toward Large-Scale Fabrication of Nanowire Devices. Small 11(40): 5402-5408.

2. Gao F, Wen L, Xu Z, Han J, Yu Y, et al. (2017) Growth of InN Nanowires with Uniform Diameter on Si(111) Substrates: Competition Between Migration and Desorption of In Atoms. Small 13(21).

3. Kwon SY, Moon SK, Lee CW, Ebaid M, Kang JH, et al. (2016) Optical Characteristics of Single-Crystal InGaN Nanowires Grown via MetalOrganic Chemical Vapor Deposition with a Ni Catalyst. J Nanosci Nanotechnol 16(12): 12967-12970.

4. Ha R, Kim SW, Choi HJ (2013) Fabrication of vertical GaN/InGaN heterostructure nanowires using $\mathrm{Ni}$-Au bi-metal catalysts. Nanoscale Res Lett 8(1): 299.

5. Park YH, Ha R, Park TE, Kim SW, Seo D, et al. (2015) Magnetic In Ga1 $\mathrm{N}$ nanowires at room temperature using $\mathrm{Cu}$ dopant and annealing. Nanoscale Res Lett 10(3).

6. Tang TY, Lin CH, Chen YS, Shiao WY, Chang WM, et al. (2010) Nitride Nanocolumns for the Development of Light-Emitting Diode. IEEE T Electron Dev 57(1): 71-78.

7. Xu Z, Yu Y, Han J, Wen L, Gao F, et al. (2017) The mechanism of indiumassisted growth of (In)GaN nanorods: eliminating nanorod coalescence by indium-enhanced atomic migration. Nanoscale 9(43): 16864-16870.

8. Song J, Leung B, Zhang Y, Han J (2014) Growth, structural and optical properties of ternary InGaN nanorods prepared by selective-area metalorganic chemical vapor deposition. Nanotechnology 25(22): 225602 .

9. Shen J, Zheng Y, Xu Z, Yu Y, Gao F, et al. (2018) Crystallographic plane and topography-dependent growth of semipolar InGaN nanorods on patterned sapphire substrates by molecular beam epitaxy. Nanoscale 10(46): 21951-21959. 